\title{
Tous les jours de la vie
}

Souvenirs d'un militant du MIR chilien

\section{Enérico García Concha}

\section{(2) OpenEdition}

Journals

Édition électronique

URL : http://journals.openedition.org/conflits/18141

DOI : $10.4000 /$ conflits. 18141

ISSN : $1777-5345$

Éditeur :

CCLS - Centre d'études sur les conflits lilberté et sécurité, L'Harmattan

Édition imprimée

Date de publication : 5 septembre 2011

Pagination : 173-180

ISBN : 978-2-296-56086-4

ISSN : 1157-996X

Référence électronique

Enérico García Concha, « Tous les jours de la vie », Cultures \& Conflits [En ligne], 81-82 | Printemps/Été 2011, mis en ligne le 05 septembre 2012, consulté le 30 mars 2021. URL : http://

journals.openedition.org/conflits/18141; DOI : https://doi.org/10.4000/conflits.18141 


\title{
Tous les jours de la vie
}

\section{Souvenirs d'un militant du MIR chilien}

\section{Enérico GARCÍA CONCHA}

Note de la Rédaction.- L'ouvrage ${ }^{1}$, dont nous publions ici un extrait, aborde des épisodes de la vie politique chilienne contemporaine et, en particulier, ce que fut le militantisme au sein du Mouvement de Gauche Révolutionnaire (MIR), depuis sa formation - au milieu des années 1960 - jusqu'au milieu des années 19802.

L'auteur est un ancien militant. Il a rejoint le MIR en 1968 et a été, entre autres, membre de la première escorte présidentielle de Salvador Allende, et responsable de la sécurité de la commission politique $(C P)$ du même parti. Sans être une autobiographie stricto sensu, le livre se structure autour de ses souvenirs et de ses réflexions.

L'extrait choisi évoque la journée du coup d'État du 11 septembre 1973. Afin de ne pas surcharger le récit de notes explicatives, précisons que "Miguel " est Miguel Enríquez, que les personnes qui l'entourent sont membres de la commission politique du MIR, à l'exception d'Eduardo Ojeda, dit "León ", miriste dont les tâches sont précisées dans le texte.

\section{1 septembre 1973 - INDUMET}

\begin{abstract}
À l'époque, Andrés Pascal Allende et moi, nous vivions dans le même quar-

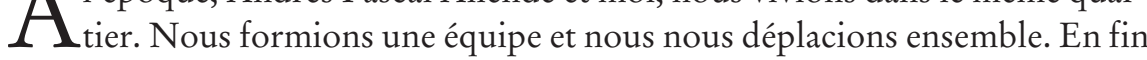
de journée, après avoir quitté la maison où se réunissait la commission politique, la voiture m'était confiée. Nous nous retrouvions tous les matins, vers huit heures.
\end{abstract}

1. Todos los días de la vida. Recuerdos de un militante del MIR Chileno, Santiago, Editorial Cuarto Propio, 2010. Extrait traduit de l'espagnol par Antonia García Castro.

2. Le MIR ne faisait pas partie de l'Unité Populaire et prônait la lutte armée là où le Président Allende, élu le 4 septembre 1970, défendait la voie démocratique vers le socialisme. Au demeurant, le MIR a apporté son soutien à plusieurs reprises au gouvernement socialiste ; notamment dès son élection, puisque la première escorte présidentielle - dite Garde des Amis Personnels, GAP - était composée de militants miristes. 
Le 11 septembre, Andrés Pascal est venu me chercher plus tôt en me disant que, d'après des sources sûres, le coup d'État avait commencé à Valparaiso. Un rendez-vous avait été fixé à sept heures et demie, dans le quartier de Gran Avenida (il y avait du côté de San Ignacio, une petite place qui formait une sorte de triangle). C'est là qu'on devait retrouver notre contact pour ensuite rejoindre le nouveau quartier général de la commission politique du MIR. On s'est mis en route. Presque toute la CP était déjà sur place. Les conversations ont alors commencé pour tenter de déterminer les prochaines actions.

Ces conversations ont surtout été téléphoniques. Miguel appelle La Moneda ${ }^{3}$ et parvient à parler avec Allende. Miguel insiste sur le fait que son discours devra d'abord viser la résistance du peuple et contenir un appel à la désobéissance des sous-officiers et des soldats vis-à-vis des commandements putschistes. C'est ce qu'il lui demande et il lui dit que nous allons chercher des manières de résister. D'autres coups de fil sont donnés aux organisations politiques sœurs. Le Parti socialiste, le MAPU ${ }^{4}$. Miguel parle avec Carlos Altamirano ${ }^{5}$ et apprend que la direction du PS va se réunir dans une petite usine située dans le secteur de Vicuña Mackenna, INDUMET ${ }^{6}$. Un rendezvous est fixé.

Miguel essaye de reparler avec le Président mais celui-ci ne prend plus les appels. C'est par l'intermédiaire de Tati ${ }^{7}$ que le Président lui envoie, néanmoins, un message : " Allende fait dire à Miguel que l'heure du MIR est arrivée, que l'heure d'un autre type de direction est arrivée ». À partir de ce moment, le contact est interrompu avec La Moneda et il n'y aura plus d'échanges. On se prépare pour la réunion à INDUMET.

Miguel, Humberto Sotomayor et moi-même, nous nous rendons à cette première réunion. Nous arrivons à INDUMET vers dix heures du matin. On y retrouve Calderón, dirigeant du Parti Socialiste, qui converge avec Miguel sur la nécessité de mettre en place un plan de résistance. Le PS avait convoqué plutôt ses secteurs elenos ${ }^{8}$. On décide de réunir les deux commandements, du MIR et du PS, afin de mettre en place une résistance commune. Une nouvelle réunion est fixée une heure et demie plus tard. Nous retournons à la maison où se trouvent les autres membres de la CP. Lesquels avaient été occupés ailleurs.

3. Palais présidentiel.

4. Mouvement d'Action Populaire Unitaire. Groupe issu d'une scission, à gauche, de la démocratie chrétienne.

5. Haut dirigeant du PS chilien.

6. Le choix du lieu a son importance. Il y avait à Santiago plusieurs secteurs où se concentraient des usines (dont celui de Vicuña Mackenna). Sous l'Unité Populaire on entreprit d'établir une coordination entre ces usines, non pas par secteur d'activité mais par zones géographiques: ce furent les « cordons industriels». Il s'agissait non seulement d'organiser la production mais aussi de mettre en place des foyers de résistance.

7. Surnom de Beatriz Allende, fille du Président Allende. 
Ainsi, Andrés Pascal et Arturo Villavela s'étaient rendus à l'ambassade cubaine pour demander la remise des armes qui s'y trouvaient, dans l'idée de pouvoir armer le plus de gens possible. Ce fut un nouveau refus, pour les mêmes motifs invoqués le 29 juin ${ }^{9}$ : cette décision ne pouvait être prise que par Salvador Allende. Et tant qu'il n'y aurait pas un ordre explicite de la part du Président visant à lancer des plans de résistance à un niveau plus global, ils ne pouvaient remettre les armes à qui que ce soit. La sortie des camarades n'est pas facile. Des civils, partisans du coup d'État, avaient bloqué la rue de l'ambassade. Ils sortent donc « à main armée ».

Il était clair désormais qu'on ne pouvait compter que sur nos propres forces. Le seul armement lourd dont on disposait était celui de la Fuerza Central ${ }^{10}$. Deux fusils sans recul $57 \mathrm{~mm}$ et deux mitrailleuses point 30 , qui se trouvaient à leur place habituelle. Il y avait aussi quelques carabines en possession de la Fuerza, et les armes de poing disséminées au sein de l'organisation. On disposait par ailleurs d'un certain armement plus circonstanciel, quelques mines, etc.

Sachant à quoi s'en tenir, la commission politique du MIR décide de résister. On se rend donc à la réunion avec les dirigeants du Parti Socialiste.

Nous sommes cinq camarades du MIR à partir : Miguel, Humberto Sotomayor, Arturo Villavela, Andrés Pascal Allende et moi. Nous partons dans deux voitures.

Sur le chemin, nous croisons une voiture du parti et nous reconnaissons le chauffeur, le camarade Eduardo Ojeda, dit «León », chef de talleres ${ }^{11}$, lequel était en mission. Miguel lui demande de s'arrêter et lui demande de nous suivre. Le camarade nous suit et se met au bout de la petite caravane. Nous arrivons à INDUMET.

\section{II}

Calderón n’y était plus. En représentation du PS, nous avons trouvé le camarade Arnoldo Camú. Il y avait aussi d'autres camarades que nous connaissions bien dont Rafael Ruiz Moscatelli.

Nous entrons dans une maison préfabriquée, dans une petite pièce où le chef d'INDUMET avait son bureau. Les membres des deux directions commencent à discuter pour déterminer le plan.

8. Militants de l'Armée de Libération Nationale Socialiste (dont les sigles en espagnol sont ELN, d'où l'appellation elenos). Il s'agissait d'une tendance (guévariste) au sein du PS.

9. Référence au 29 juin 1973. Date d'une tentative de coup d'État avortée.

10. La Force Centrale était l'une des premières structures militaires du MIR.

11. En l'occurrence des ateliers consacrés à la fabrication et à la réparation des armes. 
En définitive, l'idée était de tenter de faire sortir Allende de La Moneda. Nous pensions que c'était la seule manière d'assurer une résistance d'envergure. Tenter de faire sortir le camarade Président de La Moneda et les camarades du GAP qui s'y trouvaient, récupérer les armes, emmener Allende vers le secteur sud de Santiago, où nous avions plus de chances de le cacher, voire même, après, la possibilité de lui faire quitter le pays.

Il s'agissait de soutenir cette résistance. Il s'agissait de faire en sorte qu'Allende ne se rende pas et ne meure pas dans les combats. Et avec cela, maintenir la flamme de la résistance absolument vivante; en plus du soutien international que pouvait générer Allende en vie.

La discussion étant engagée, les carabiniers arrivent dans l'intention de faire une perquisition dans l'usine; ils avaient eu l'information selon laquelle il y avait là un foyer de résistance. C'est Rafael Ruiz Moscatelli qui parle avec eux, puis ferme la porte et transmet l'information. Très vite les coups de feu commencent.

De là où nous étions, du fait de la hauteur des murs extérieurs, il n’y avait aucune possibilité de voir l'ennemi. En conséquence, il n'y avait pas d'affrontement direct, les coups de feu étaient pratiquement tirés en l'air. À ce moment là, Miguel essaye de faire pression sur la direction du Parti Socialiste afin de faire sortir l'ensemble des camarades présents à INDUMET. Il s'agissait de maintenir la cohésion du groupe. Miguel disait qu'il fallait ouvrir l'entrée principale et utiliser les voitures pour nous protéger et quitter les lieux.

Cela voulait dire sortir en un groupe compact, récupérer les voitures et mener à bien le grand projet qui était d'arriver jusqu'au centre. Aller chercher Allende à La Moneda.

La prise de décision se dilue et Miguel dit : «si on ne sort pas tout de suite, ils vont nous encercler ». Ce qui voulait dire, vu les caractéristiques du quartier, qu'on allait tous se faire tuer.

Nous n'avions que des pistolets, nos armes personnelles, les quatre membres de la CP et moi. Le camarade "León » n'était pas armé. Le camarade Andrés Pascal m'avait passé son arme (celle-là même qu'on mentionne dans plusieurs récits comme un cadeau qu'Allende avait fait à son neveu). En conséquence, Miguel demande à Arnoldo Camú de nous fournir des fusils pour pouvoir nous protéger et combattre dans de meilleures conditions. Camú donne l'ordre à cinq camarades de nous donner leurs armes.

Je m'approche d'un camarade qui me donne son fusil AK avec deux chargeurs, et il me demande de lui dire mon nom pour pouvoir le récupérer plus 
tard. Chose que j'ai trouvé assez amusante car les possibilités que nous avions de nous revoir étaient minces.

Une fois les armes en notre possession, je me souviens qu'on a considéré la possibilité de mener un affrontement de l'intérieur. Je revois Andrés Pascal et Villavela en train de monter des escaliers, je les suis et en arrivant tout en haut, nous nous rendons compte que nous sommes complètement enfermés, qu'il n'y a aucune vision sur l'extérieur. On ne voit aucun ennemi à affronter. Et quand nous redescendons, Miguel avait pris la décision de sortir, de briser l'encerclement avec nos seules forces.

Le camarade «León » n’avait pas bougé de sa voiture, garée à l’intérieur de l'enceinte. Il se déplaçait ces jours-là dans une Fiat 125 jaune, que le parti lui avait assignée et qui était dans de meilleures conditions que les autres. «León » était resté au volant de cette voiture.

\section{III}

Je voudrais faire une remarque absolument personnelle. Pendant des années, nous tous, nous avions pensé à l'affrontement, mais il était toujours hypothétique. Et soudain ce fut «maintenant ». L'affrontement avec l'ennemi commençait à la minute suivante. Je me souviens de m’être appuyé sur un mur et d'avoir pensé : «bon, nous y sommes, c'est peut-être pour cela qu’on se préparait et c'est maintenant qu'il va falloir montrer que nous sommes capables de gagner ». La grande douleur de ce moment, ça a été la possibilité de mourir et de ne pas voir grandir ma fille.

On se lance vers la partie arrière de l'usine. J'étais allé prévenir le camarade «León » qu'on quittait les lieux; et nous avions sauté vers une petite usine qui donnait sur la rue San Joaquín. Là, on se retrouve en face d'un gardien et d'une Fiat 600, on tente de récupérer les clés, il nous dit qu'il ne les a pas, qu'il n'a même pas les clés de la porte. On sort.

Rue San Joaquín, on se met à courir en direction de Vicuña Mackenna. Miguel s'était détaché, il était presque sur le bord du trottoir. Humberto Sotomayor était plus ou moins au centre et moi, du côté du mur des usines. Derrière, Villavela et Andrés Pascal. Quand on arrive au coin de la rue, je vois que Miguel lâche une rafale. Humberto Sotomayor vient en renfort, et je vois tomber deux carabiniers en plein coin de rue. Le premier coin de rue, disons. Ils restent au sol, et je me poste face à cette rue, pour assurer une protection et éviter qu'on tire sur la rue San Joaquín. Je vois passer Miguel, Humberto Sotomayor, Andrés Pascal et Villavela. Quand les quatre membres de la Commission Politique sont passés, je décide de passer à mon tour et on court, toujours rue San Joaquín, jusqu'à ce qu'on arrive devant l'usine Comandari. 
Depuis l'usine, j'entends des cris «il est touché, il est touché ». Je me retourne, en supposant que la personne qui doit venir derrière moi est le camarade «León ». Je me retourne, et je vois quelqu'un par terre, et ma première intention est d'aller le récupérer. Mais le camarade se relève et avance vers nous. Sur le coup, je ne suis pas certain que ce soit «León » mais je me calme en pensant que ce n'est qu'une chute et non que le camarade a reçu une balle. Je n'entendais pas les coups de feu, je n'entendais pas qu'on tirait directement sur nous. Alors je ne fais plus attention et je continue à courir. En arrivant à l'usine Comandari, Humberto Sotomayor décide d'y entrer et je le suis parce qu'il y avait des voitures. Il fallait récupérer une voiture pour sortir de la zone des affrontements. Nous n'avons aucune réponse de la part des gens qui se trouvaient là, tranquillement, en train d'observer le déroulement de la scène. On ne peut pas récupérer une voiture, on ressort par une porte latérale.

C'est là qu'on retrouve Miguel qui venait par l'extérieur de Comandari. Et aussi Andrés Pascal, et on continue à courir ensemble. Je me rends compte que dans le groupe il y a un garçon socialiste, un garçon assez jeune, qui avait décidé de sortir avec nous. On se retrouve dans les rues du bidonville. Tous ces bidonvilles sont rattachés aux usines du secteur. On les traverse jusqu'à ce qu'on arrive dans la zone des bâtiments, près de Vicuña Mackenna. Il y avait là une Peugeot rouge. Les gens, nous voyant armés, se sentaient immédiatement intimidés. On demande où se trouve le propriétaire de la Peugeot, on nous indique un bâtiment. C'était un appartement au deuxième étage, on entre, le propriétaire nous donne les clés sur le champ. On monte et on sort du quartier, en prenant les précautions nécessaires pour ne pas nous retrouver en présence des forces ennemies.

On finit par sortir de la zone sans essuyer de pertes. Il y a bien quelques affrontements sur le chemin, sans répercussions. C'était les gens du Sixième Commissariat situé dans le quartier de Gran Avenida. Il n'y a qu'un échange de coups de feu. Je ne sais pas si nous avons produit des blessés, le fait est que nous avons réussi à rejoindre la maison de la CP.

\section{IV}

$\mathrm{La} \mathrm{CP}$ se réunit et on prend les décisions qui vont régir par la suite. Il est clair que nous n'avons aucune possibilité de mener une résistance commune avec une autre organisation de gauche et que nous ne pouvons compter que sur nos propres forces. On décide d'appeler à un retrait stratégique et de commencer la construction d'un autre type de résistance. La Fuerza Central est mise en état d'alerte. La commission politique passe immédiatement à la clandestinité.

C'est alors que nous parviennent les premières informations relatives aux caractéristiques sanglantes du coup d'État, il devient clair que l'idée des sec- 
teurs putschistes est d'annihiler l'avant-garde du mouvement ouvrier. Nous apprenons la mort d'Allende, la prise de La Moneda, l'arrestation des camarades du GAP. Les foyers de résistance disparaissent rapidement après les premiers combats avec les forces ennemies.

Par la suite, nous avons su que les personnes restées à INDUMET avaient réussi à briser l'encerclement. Mais elles avaient ensuite rejoint le bidonville, La Legua, où elles avaient combattu pendant des jours. Plusieurs camarades ont été blessés, tués ou arrêtés.

De notre côté, nous avons été durement touchés par la mort du camarade «León ». Lequel finalement ne réussit pas à sortir d'INDUMET. D’après des récits qu'on nous a fait plus tard, il semblerait que le camarade "León » ait été blessé dans sa fuite. Dans l'impossibilité de marcher, car il était blessé à une jambe, il est retourné dans l'usine où il a, plus tard, été abattu de sang froid.

\section{9 septembre 1973}

La maison de sécurité où j'arrive le 13 septembre constituait une excellente cachette. Elle était située dans un quartier de villas, où les propriétaires étaient majoritairement des gens de droite qui ne seraient probablement pas inquiétés puisqu'ils étaient partisans du coup d'État. En conséquence, du point de vue de la sécurité, c'était une bonne maison. Mais c'était contraignant. Les accès y étaient tout de même surveillés et on était facilement contrôlé sur la route. Cela m'empêchait de me déplacer et de chercher une connexion avec le parti pour commencer à travailler de manière organique, dans ce qui allait se décider.

La question de la sécurité de la commission politique cessait d'être importante. Ou, plutôt, les structures de sécurité devaient être remontées d'une manière différente par rapport à celle qui avait prévalue jusqu'à ce moment-là. Les équipes de sécurité devaient être plus petites. Il était parfois plus facile de déplacer un seul membre de la $\mathrm{CP}$, ou deux membres de la CP, qui se protégeaient mutuellement, que d'avoir un appareil de sécurité assigné à cette tâche.

Je pense que ma décision de quitter cette maison a été trop rapide. J'aurais dû attendre davantage. Le fait de l'abandonner me mettait dans une situation de fragilité assez sévère. Je commence alors à vivre une série de situations compliquées, comme le fait d'arriver dans une maison quelconque, cinq minutes avant le couvre-feu, demander l'autorisation d'y rester, essuyer un refus, et devoir poser mon arme sur la table en disant : « je ne bouge pas d'ici avant huit heures du matin ». Et ne pouvoir fermer l'œil par crainte d'être dénoncé.

Chaque jour qui passait était une odyssée. Je savais que ma propre maison allait être perquisitionnée. Un cadet de l'école militaire (qui a par la suite tra- 
vaillé avec les services de renseignements, et que nous avions pour voisin) avait transmis cette information à ma mère : la perquisition avait été ordonnée pour le 11 septembre, au soir. À la dernière minute, une autre opération, de plus grande importance pour eux, était survenue. C'était la possibilité d'arrêter un haut dirigeant de la commission politique du Parti Communiste. La perquisition à mon domicile a donc été remise à plus tard.

Au final, je réussis à tenir jusqu'au 29 septembre. Le 29 septembre, la maison où je logeais fait l'objet d'une descente des Carabiniers, opération conduite par le capitaine Esquivel. Je suis arrêté. 